\title{
DIFFERENCE EQUATIONS WITH SEVERAL NON-MONOTONE DEVIATING ARGUMENTS: ITERATIVE OSCILLATION TESTS
}

\author{
GEORGE E. CHATZARAKIS ${ }^{1}$ AND IRENA JADLOVSKÁ ${ }^{2}$ \\ ${ }^{1}$ Department of Electrical and Electronic Engineering Educators \\ School of Pedagogical and Technological Education (ASPETE) \\ 14121, N. Heraklio, Athens, GREECE \\ ${ }^{2}$ Departmens of Mathematics and Theoretical Informatics \\ Faculty of Electrical Engineering and Informatics \\ Technical University of Košice \\ Letná 9, 04200 Košice, SLOVAKIA
}

\begin{abstract}
This paper is a study on the oscillatory behavior of the solutions of a difference equation with several non-monotone deviating arguments and nonnegative coefficients. New sufficient oscillation conditions involving limsup are established using an iterative method. The conditions obtained by this method achieve a marked improvement on all known conditions, requiring at the same time far fewer iterations to determine whether all solutions of the considered equations oscillate, than the other known conditions. Examples, numerically solved in MATLAB, are provided to illustrate the results and the improvement achieved.
\end{abstract}

AMS Subject Classification: 39A10, 39A21

Key Words: difference equation, non-monotone arguments, oscillatory solutions, nonoscillatory solutions, Grönwall inequality

Received: January 13, 2018; Accepted: February 18, 2018;

Published: March 11, $2018 \quad$ doi: $10.12732 /$ dsa.v27i2.5

Dynamic Publishers, Inc., Acad. Publishers, Ltd. https://acadsol.eu/dsa

\section{INTRODUCTION}

In this paper we consider the first-order linear difference equation with variable re- 
tarded arguments of the form

$$
\Delta x(n)+\sum_{i=1}^{m} p_{i}(n) x\left(\tau_{i}(n)\right)=0, \quad n \in \mathbb{N}_{0},
$$

and the (dual) difference equation with variable advanced arguments of the form

$$
\nabla x(n)-\sum_{i=1}^{m} q_{i}(n) x\left(\sigma_{i}(n)\right)=0, \quad n \in \mathbb{N},
$$

where $\left(p_{i}(n)\right)_{n \geq 0},\left(q_{i}(n)\right)_{n \geq 1}, 1 \leq i \leq m$, are sequences of nonnegative real numbers, and $\left(\tau_{i}(n)\right)_{n \geq 0},\left(\sigma_{i}(n)\right)_{n \geq 1}, 1 \leq i \leq m$, are sequences of integers such that

$$
\tau_{i}(n) \leq n-1, \quad \forall n \in \mathbb{N}_{0} \quad \text { and } \quad \lim _{n \rightarrow \infty} \tau_{i}(n)=\infty, \quad 1 \leq i \leq m
$$

and

$$
\sigma_{i}(n) \geq n+1, \quad \forall n \in \mathbb{N}, \quad 1 \leq i \leq m,
$$

respectively.

Here $\mathbb{N}_{0}, \mathbb{N}$ are the sets of nonnegative integers and positive integers, respectively, $\Delta$ denotes the forward difference operator $\Delta x(n)=x(n+1)-x(n)$ and $\nabla$ corresponds to the backward difference operator $\nabla x(n)=x(n)-x(n-1)$.

Set $v=-\min \underset{\substack{n \geq 0 \\ 1 \leq i \leq m}}{ } \tau_{i}(n)$ and note that $v$ is a finite positive integer, if (1.1) holds.

By a solution of $(E)$, we mean a sequence of real numbers $(x(n))_{n \geq-v}$ which satisfies $(E)$, for all $n \geq 0$. It is clear that, for each choice of real numbers $c_{-v}$, $c_{-v+1}, \ldots, c_{-1}, c_{0}$, there exists a unique solution $(x(n))_{n \geq-v}$ of $(E)$ which satisfies the initial conditions $x(-v)=c_{-v}, x(-v+1)=c_{-v+1}, \ldots, x(-1)=c_{-1}, x(0)=c_{0}$. When the initial data are given, we can obtain a unique solution to $(E)$ by using the method of steps.

By a solution of $\left(E^{\prime}\right)$, we mean a sequence of real numbers $(x(n))_{n \geq 0}$ which satisfies $\left(E^{\prime}\right)$ for all $n \geq 1$.

A solution $(x(n))_{n \geq-v}\left(\right.$ or $\left.(x(n))_{n \geq 0}\right)$ of $(E)$ (or $\left.\left(E^{\prime}\right)\right)$ is called oscillatory, if the terms $x(n)$ of the sequence are neither eventually positive nor eventually negative. Otherwise, the solution is said to be nonoscillatory.

While deviating difference equations with one argument have been studied widely and extensively by several researchers, the study of such equations, especially systems involving several arguments, is scarce and rare, most likely due to the complexity of the analysis of those equations and lack of an established theory. However, recent studies in biological, physical and economics systems, involving multiple feedback mechanisms have stimulated interest on equations $(E)$ and $\left(E^{\prime}\right)$. Hence, in the last few decades, the oscillatory behavior, stability and existence of positive solutions of equations $(E)$ and $\left(E^{\prime}\right)$ has been the subject of several studies. See, for example, 
[1-18] and the references cited therein. Most of these papers though, are concerned with the special case where the arguments are nondecreasing, while merely a small number of papers are dealing with the general case where the arguments are not necessarily monotone, see, for example, [2-4].

The motivation for considering non-monotone arguments is not of purely mathematical interest. Several phenomena require the use of non-monotone arguments since there are always natural disturbances, e.g. noise in communication systems, that affect all the parameters of an equation. Therefore, the monotone arguments, adequate from a mathematical point of view, become non-monotone almost always. In view of this, an interesting question arising in the case when the arguments $\tau_{i}(n)$ and $\sigma_{i}(n)$ are non-monotone, is whether we can establish oscillation criteria that substantially improve on all the known results in the literature. This paper offers an affirmative answer to this question.

The organization will be as follows. First, we present, separately for a delay and advanced case, a short chronological review of the most interesting oscillation conditions for the above equations. Next, we establish new sufficient conditions of limsup type, for the oscillation of all solutions of $(E)$ and $\left(E^{\prime}\right)$. We base our technique on the proper use of a recursive procedure leading to new inequalities which may replace former ones. To verify the significance of the obtained results, we provide two examples along with various comparisons among new and known criteria.

Throughout this paper, we are going to use the following notation:

$$
\begin{gathered}
\sum_{i=k}^{k-1} A(i)=0 \quad \text { and } \prod_{i=k}^{k-1} A(i)=1 \\
\alpha:=\liminf _{n \rightarrow \infty} \sum_{i=1}^{m} \sum_{j=\tau(n)}^{n-1} p_{i}(j) \\
\beta:=\liminf _{n \rightarrow \infty} \sum_{i=1}^{m} \sum_{j=n+1}^{\sigma(n)} q_{i}(j) \\
\quad \begin{array}{l}
0, \\
\quad(\omega):=\limsup _{n \rightarrow \infty} \sum_{i=1}^{m} \sum_{j=\tau(n)}^{n} p_{i}(j) \\
M A:=\limsup _{n \rightarrow \infty} \sum_{i=1}^{m} \sum_{j=n}^{\sigma(n)} q_{i}(j)
\end{array}
\end{gathered}
$$

where $\tau(n)=\max _{1 \leq i \leq m} \tau_{i}(n), \sigma(n)=\min _{1 \leq i \leq m} \sigma_{i}(n)$ and $\tau_{i}(n), \sigma_{i}(n)$ are nondecreasing. 


\subsection{RETARDED DIFFERENCE EQUATIONS (CHRONOLOGICAL REVIEW)}

In 2006, Berezansky and Braverman [1] and in 2014, Chatzarakis, Pinelas and Stavroulakis [8] proved that if

$$
\limsup _{n \rightarrow \infty} \sum_{i=1}^{m} p_{i}(n)>0 \quad \text { and } \quad \alpha>\frac{1}{e},
$$

or

$$
M D>1
$$

respectively, then all solutions of $(E)$ are oscillatory.

Now let us come to the case considered in the present work, i.e., that the arguments $\tau_{i}(n), 1 \leq i \leq m$ are not necessarily monotone.

Set

$$
h(n)=\max _{1 \leq i \leq m} h_{i}(n) \quad \text { where } \quad h_{i}(n)=\max _{0 \leq s \leq n} \tau_{i}(s), \quad n \geq 0
$$

and

$$
\begin{gathered}
a_{1}(n, k):=\prod_{i=k}^{n-1}\left[1-\sum_{\ell=1}^{m} p_{\ell}(i)\right] \\
a_{r+1}(n, k):=\prod_{i=k}^{n-1}\left[1-\sum_{\ell=1}^{m} p_{\ell}(i) a_{r}^{-1}\left(i, \tau_{\ell}(i)\right)\right], \quad r \in \mathbb{N} .
\end{gathered}
$$

Clearly, $h_{i}(n), h(n)$ are nondecreasing and $\tau_{i}(n) \leq h_{i}(n) \leq h(n) \leq n-1$, for all $n \geq 0$.

In 2015, Braverman, Chatzarakis and Stavroulakis [2] proved that if there exists a subsequence $\theta(n), n \in \mathbb{N}$ of positive integers such that

$$
\sum_{i=1}^{m} p_{i}(\theta(n)) \geq 1, \quad \forall n \in \mathbb{N},
$$

then all solutions of $(E)$ are oscillatory.

Under the assumption that

$$
\sum_{i=1}^{m} p_{i}(n)<1, \quad \forall n \geq 0,
$$

the same authors proved that, if for some $r \in \mathbb{N}$

$$
\limsup _{n \rightarrow \infty} \sum_{j=h(n)}^{n} \sum_{i=1}^{m} p_{i}(j) a_{r}^{-1}\left(h(n), \tau_{i}(j)\right)>1,
$$

then all solutions of $(E)$ are oscillatory. 
In 2017, Chatzarakis, Horvat-Dmitrović and Pašić [3] proved that if, for some $\ell \in \mathbb{N}$

$$
\limsup _{n \rightarrow \infty} \sum_{j=h(n)}^{n} \mathcal{P}(j) \prod_{i=\tau(j)}^{h(n)-1} \frac{1}{1-\mathcal{P}_{\ell}(i)}>1
$$

where

$$
\mathcal{P}_{\ell}(n)=\mathcal{P}(n)\left[1+\sum_{i=\tau(n)}^{n-1} \mathcal{P}(i) \prod_{j=\tau(i)}^{h(n)-1} \frac{1}{1-\mathcal{P}_{\ell-1}(j)}\right]
$$

with $\mathcal{P}(n)=\mathcal{P}_{0}(n)=\sum_{i=1}^{m} p_{i}(n)$, then all solutions of $(E)$ are oscillatory.

Lately, Chatzarakis and Jadlovská [4] proved that if, for some $w \in \mathbb{N}$,

$$
\limsup _{n \rightarrow \infty} \sum_{\ell=h(n)}^{n} \bar{P}(\ell) \prod_{i=\tau(\ell)}^{h(n)-1} \frac{1}{1-\bar{P}_{w}(i)}>1
$$

where

$$
\bar{P}_{w}(n)=\bar{P}(n)\left[1+\sum_{\ell=\tau(n)}^{n-1} \bar{P}(\ell) \exp \left(\sum_{j=\tau(\ell)}^{n-1} \bar{P}(j) \prod_{i=\tau(j)}^{j-1} \frac{1}{1-\bar{P}_{w-1}(i)}\right)\right],
$$

with $\bar{P}(n)=\bar{P}_{0}(n)=\sum_{i=1}^{m} p_{i}(n)$, then all solutions of $(E)$ are oscillatory.

\subsection{ADVANCED DIFFERENCE EQUATIONS (CHRONOLOGICAL REVIEW)}

In 2014, Chatzarakis, Pinelas and Stavroulakis [8] proved that if

$$
M A>1,
$$

then all solutions of $\left(E^{\prime}\right)$ are oscillatory.

Assume that the arguments $\sigma_{i}(n), 1 \leq i \leq m$, are not necessarily monotone.

Set

$$
\rho(n)=\min _{1 \leq i \leq m} \rho_{i}(n), \quad \text { where } \rho_{i}(n)=\min _{s \geq n} \sigma_{i}(s), \quad n \geq 0
$$

and

$$
\begin{array}{cc}
b_{1}(n, k):=\prod_{i=n+1}^{k}\left[1-\sum_{\ell=1}^{m} q_{\ell}(i)\right], & \\
b_{r+1}(n, k):=\prod_{i=n+1}^{k}\left[1-\sum_{\ell=1}^{m} q_{\ell}(i) b_{r}^{-1}\left(i, \sigma_{\ell}(i)\right)\right], \quad r \in \mathbb{N} .
\end{array}
$$

Clearly, $\rho_{i}(n), \rho(n)$ are nondecreasing and $\sigma_{i}(n) \geq \rho_{i}(n) \geq \rho(n) \geq n+1$ for all $n \geq 1$. 
In 2015, Braverman, Chatzarakis and Stavroulakis [2] proved that if there exists a subsequence $\theta(n), n \in \mathbb{N}$ of positive integers such that

$$
\sum_{i=1}^{m} q_{i}(\theta(n)) \geq 1, \quad \forall n \in \mathbb{N},
$$

then all solutions of $\left(E^{\prime}\right)$ are oscillatory.

Under the assumption that

$$
\sum_{i=1}^{m} q_{i}(n)<1, \quad \forall n \geq 1
$$

the same authors proved that, if for some $r \in \mathbb{N}$,

$$
\limsup _{n \rightarrow \infty} \sum_{j=n}^{\rho(n)} \sum_{i=1}^{m} q_{i}(j) b_{r}^{-1}\left(\rho(n), \sigma_{i}(j)\right)>1,
$$

then all solutions of $\left(E^{\prime}\right)$ are oscillatory.

Recently, Chatzarakis, Horvat-Dmitrović and Pašić [3] proved that if for some $\ell \in \mathbb{N}$

$$
\limsup _{n \rightarrow \infty} \sum_{j=n}^{\rho(n)} Q(j) \prod_{i=\rho(n)+1}^{\sigma(j)} \frac{1}{1-Q_{\ell}(i)}>1
$$

where

$$
Q_{\ell}(n)=Q(n)\left[1+\sum_{i=n+1}^{\rho(n)} Q(i) \prod_{j=\rho(n)+1}^{\sigma(i)} \frac{1}{1-Q_{\ell-1}(j)}\right]
$$

with $Q(n)=Q_{0}(n)=\sum_{i=1}^{m} q_{i}(n)$, then all solutions of $\left(E^{\prime}\right)$ are oscillatory.

Lately, Chatzarakis and Jadlovská [4] proved that, if for some $w \in \mathbb{N}$

$$
\limsup _{n \rightarrow \infty} \sum_{\ell=n}^{\rho(n)} \bar{Q}(\ell) \prod_{i=\rho(n)+1}^{\sigma(\ell)} \frac{1}{1-\bar{Q}_{w}(i)}>1
$$

where

$$
\bar{Q}_{w}(n)=\bar{Q}(n)\left[1+\sum_{\ell=n+1}^{\sigma(n)} \bar{Q}(\ell) \exp \left(\sum_{j=n+1}^{\sigma(\ell)} \bar{Q}(j) \prod_{i=j+1}^{\sigma(j)} \frac{1}{1-\bar{Q}_{w-1}(i)}\right)\right],
$$

with $\bar{Q}(n)=\sum_{i=1}^{m} q_{i}(n)=\bar{Q}_{0}(n)$, then all solutions of $\left(E^{\prime}\right)$ are oscillatory. 


\section{BASIC LEMMAS}

\subsection{RETARDED DIFFERENCE EQUATIONS}

The proofs of our main results are essentially based on the following lemmas.

Lemma 1. Assume that (1.1) holds and $\alpha$ is defined by (1.3) with $\alpha>0$. Then we have

$$
\liminf _{n \rightarrow \infty} \sum_{i=1}^{m} \sum_{j=h(n)}^{n-1} p_{i}(j)=\liminf _{n \rightarrow \infty} \sum_{i=1}^{m} \sum_{j=\tau(n)}^{n-1} p_{i}(j)=\alpha,
$$

where $h(n)$ is defined by (1.7) and $\tau(n)=\max _{1 \leq i \leq m} \tau_{i}(n)$.

Proof. Clearly, the sequence of integers $h(n)$ is nondecreasing and $\tau(n) \leq h(n) \leq$ $n-1$, for all $n \geq 0$. So

$$
\sum_{i=1}^{m} \sum_{j=h(n)}^{n-1} p_{i}(j) \leq \sum_{i=1}^{m} \sum_{j=\tau(n)}^{n-1} p_{i}(j)
$$

Hence

$$
\liminf _{n \rightarrow \infty} \sum_{i=1}^{m} \sum_{j=h(n)}^{n-1} p_{i}(j) \leq \liminf _{n \rightarrow \infty} \sum_{i=1}^{m} \sum_{j=\tau(n)}^{n-1} p_{i}(j) .
$$

Assuming that (2.1) does not hold, there exists $\alpha^{\prime}>0$ and a subsequence $(\theta(n))$ such that $\theta(n) \rightarrow \infty$ as $n \rightarrow \infty$ and

$$
\lim _{n \rightarrow \infty} \sum_{i=1}^{m} \sum_{j=h(\theta(n))}^{\theta(n)-1} p_{i}(j) \leq \alpha^{\prime}<\alpha .
$$

By definition, $h(\theta(n))=\max _{1 \leq i \leq m} h_{i}(\theta(n))$, where $h_{i}(\theta(n))=\max _{0 \leq s \leq \theta(n)} \tau_{i}(s)$. It is obvious that there is $\ell \in\{1, \ldots, m\}$ such that

$$
h(\theta(n))=h_{\ell}(\theta(n)) \quad \text { and } \quad h_{j}(\theta(n)) \leq h_{\ell}(\theta(n)), \quad j \in\{1, \ldots, m\} .
$$

But $h_{\ell}(\theta(n))=\max _{0 \leq s \leq \theta(n)} \tau_{\ell}(s)$, hence there exists $\theta^{\prime}(n) \leq \theta(n), \theta^{\prime}(n) \in \mathbb{N}_{0}$ such that $h_{\ell}(\theta(n))=\tau_{\ell}\left(\theta^{\prime}(n)\right)$. Due to $\tau\left(\theta^{\prime}(n)\right)=\max _{1 \leq i \leq m} \tau_{i}\left(\theta^{\prime}(n)\right)$, we have

$$
\tau\left(\theta^{\prime}(n)\right) \geq \tau_{\ell}\left(\theta^{\prime}(n)\right)=h_{\ell}(\theta(n))=h(\theta(n))
$$

and consequently

$$
\sum_{i=1}^{m} \sum_{j=h(\theta(n))}^{\theta(n)-1} p_{i}(j) \geq \sum_{i=1}^{m} \sum_{j=\tau\left(\theta^{\prime}(n)\right)}^{\theta(n)-1} p_{i}(j) \geq \sum_{i=1}^{m} \sum_{j=\tau\left(\theta^{\prime}(n)\right)}^{\theta^{\prime}(n)-1} p_{i}(j) .
$$


It follows that $\left(\sum_{i=1}^{m} \sum_{j=\tau\left(\theta^{\prime}(n)\right)}^{\theta^{\prime}(n)-1} p_{i}(j)\right)_{n=1}^{\infty}$ is a bounded sequence, having a convergent subsequence, say

$$
\sum_{i=1}^{m} \sum_{j=\tau\left(\theta^{\prime}\left(n_{k}\right)\right)}^{\theta^{\prime}\left(n_{k}\right)-1} p_{i}(j) \rightarrow c \leq \alpha^{\prime}, \quad \text { as } k \rightarrow \infty
$$

which implies that

$$
\liminf _{n \rightarrow \infty} \sum_{i=1}^{m} \sum_{j=\tau(n)}^{n-1} p_{i}(j) \leq \alpha^{\prime}<\alpha .
$$

This contradicts (1.3).

The proof of the lemma is complete.

Lemma 2. Assume that (1.1) holds, $\alpha$ is defined by (1.3) with $0<\alpha \leq 1 / e$, and $x(n)$ is an eventually positive solution of $(E)$. Then we have

$$
\liminf _{n \rightarrow \infty} \frac{x(h(n))}{x(n)} \geq \lambda_{0}
$$

where $h(n)$ is defined by (1.7) and $\lambda_{0}$ is the smaller root of the transcendental equation $\lambda=e^{\alpha \lambda}$.

Proof. Assume that $(x(n))_{n \geq-w}$ is an eventually positive solution of $(E)$. Then there exists $n_{1} \geq-w$ such that $x(n), x\left(\tau_{i}(n)\right)>0$, for all $n \geq n_{1}$. In view of this, Eq. (E) becomes

$$
\Delta x(n)=-\sum_{i=1}^{m} p_{i}(n) x\left(\tau_{i}(n)\right) \leq 0, \quad \forall n \geq n_{1},
$$

which means that $(x(n))$ is an eventually nonincreasing sequence of positive numbers.

By the definition of $\alpha$ and using Lemma 1, it is clear that there exists $\varepsilon \in(0, \alpha)$ such that

$$
\sum_{i=1}^{m} \sum_{j=h(n)}^{n-1} p_{i}(j) \geq \alpha-\varepsilon \quad \text { for } n \geq n(\varepsilon) \geq n_{1} .
$$

We will show that

$$
\liminf _{n \rightarrow \infty} \frac{x(h(n))}{x(n)} \geq \lambda_{0}(\varepsilon),
$$

where $\lambda_{0}(\varepsilon)$ is the smaller root of the equation

$$
e^{(\alpha-\varepsilon) \lambda}=\lambda
$$

Assume, for the sake of contradiction, that (2.3) is not valid. Then, there exists $\varepsilon_{0}>0$ such that

$$
\frac{e^{(\alpha-\varepsilon) \gamma}}{\gamma} \geq 1+\varepsilon_{0},
$$


where

$$
\gamma=\liminf _{n \rightarrow \infty} \frac{x(h(n))}{x(n)}<\lambda_{0}(\varepsilon)
$$

On the other hand, for any $\delta>0$, there exists $n(\delta)$ such that

$$
\frac{x(h(n))}{x(n)} \geq \gamma-\delta \quad \text { for } n \geq n(\delta) .
$$

Dividing $(E)$ by $x(n)$, we obtain

$$
\begin{aligned}
\frac{\Delta x(n)}{x(n)} & =-\sum_{i=1}^{m} p_{i}(n) \frac{x\left(\tau_{i}(n)\right)}{x(n)} \\
& \leq-\frac{x(h(n))}{x(n)} \sum_{i=1}^{m} p_{i}(n) \leq-(\gamma-\delta) \sum_{i=1}^{m} p_{i}(n)
\end{aligned}
$$

or

$$
\frac{\Delta x(n)}{x(n)} \leq-(\gamma-\delta) \sum_{i=1}^{m} p_{i}(n)
$$

Summing up the last inequality from $h(n)$ to $n-1$, we take

$$
\sum_{j=h(n)}^{n-1} \frac{\Delta x(j)}{x(j)} \leq-(\gamma-\delta) \sum_{j=h(n)}^{n-1} \sum_{i=1}^{m} p_{i}(j) \leq-(\gamma-\delta)(\alpha-\varepsilon) .
$$

But, since $e^{x} \geq x+1, \forall x>0$, we have

$$
\begin{aligned}
\sum_{j=h(n)}^{n-1} \frac{\Delta x(j)}{x(j)} & =\sum_{j=h(n)}^{n-1}\left(\frac{x(j+1)}{x(j)}-1\right) \\
& =\sum_{j=h(n)}^{n-1} \exp \left(\ln \frac{x(j+1)}{x(j)}\right)-(n-h(n)) \\
& \geq \sum_{j=h(n)}^{n-1}\left(1+\ln \frac{x(j+1)}{x(j)}\right)-(n-h(n)) \\
& =(n-h(n))+\sum_{j=h(n)}^{n-1} \ln \frac{x(j+1)}{x(j)}-(n-h(n)) \\
& =\sum_{j=h(n)}^{n-1} \ln \frac{x(j+1)}{x(j)}=\ln \frac{x(n)}{x(h(n))},
\end{aligned}
$$

or

$$
\sum_{j=h(n)}^{n-1} \frac{\Delta x(j)}{x(j)} \geq \ln \frac{x(n)}{x(h(n))} .
$$

Combining (2.7) and (2.8), we have

$$
\ln \frac{x(n)}{x(h(n))} \leq-(\gamma-\delta)(\alpha-\varepsilon)
$$


i.e.,

$$
\frac{x(h(n))}{x(n)} \geq e^{(\gamma-\delta)(\alpha-\varepsilon)} \quad \text { for large } n .
$$

Therefore,

$$
\gamma=\liminf _{n \rightarrow \infty} \frac{x(h(n))}{x(n)} \geq e^{(\gamma-\delta)(\alpha-\varepsilon)},
$$

which, as $\delta \rightarrow 0$, implies

$$
\gamma \geq e^{\gamma(\alpha-\varepsilon)} .
$$

Combining the last inequality with (2.4), we obtain

$$
\frac{e^{\gamma(\alpha-\varepsilon)}}{1+\varepsilon_{0}} \geq e^{\gamma(\alpha-\varepsilon)}
$$

which is not valid, since $\varepsilon_{0}>0$. Therefore (2.3) is true. Since $\lambda_{0}(\varepsilon) \rightarrow \lambda_{0}$ as $\varepsilon \rightarrow 0$, (2.3) implies (2.2).

The proof of the lemma is complete.

The next lemma provides a lower estimate for the ratio $x(n+1) / x(h(n))$, in terms of the smaller root of $d^{2}-(1-\alpha) d+\alpha^{2} / 2=0$. The proof of this lemma is similar to the proof of Lemma 2.1 in [7].

Lemma 3. Assume that (1.1) holds, $h(n)$ is defined by (1.7), $0<\alpha \leq 1 /$ e and $x(n)$ is an eventually positive solution of $(E)$. Then

$$
\liminf _{n \rightarrow \infty} \frac{x(n+1)}{x(h(n))} \geq D(\alpha) .
$$

\subsection{ADVANCED DIFFERENCE EQUATIONS}

Similar lemmas for the (dual) advanced difference equation $\left(E^{\prime}\right)$, easily, can be derived. The proofs of these lemmas are omitted, since they are quite similar to those of the corresponding lemmas, for the retarded equation.

Lemma 4. Assume that (1.2) holds and $\beta$ is defined by (1.4) with $\beta>0$. Then we have

$$
\liminf _{n \rightarrow \infty} \sum_{i=1}^{m} \sum_{j=n+1}^{\rho(n)} q_{i}(j)=\liminf _{n \rightarrow \infty} \sum_{i=1}^{m} \sum_{j=n+1}^{\sigma(n)} q_{i}(j)=\beta,
$$

where $\rho(n)$ is defined by (1.15) and $\sigma(n)=\min _{1 \leq i \leq m} \sigma_{i}(n)$.

Lemma 5. Assume that (1.2) holds, $\beta$ is defined by (1.4) with $0<\beta \leq 1 / e$, and $x(n)$ is an eventually positive solution of $\left(E^{\prime}\right)$. Then we have

$$
\liminf _{n \rightarrow \infty} \frac{x(\rho(n))}{x(n)} \geq \lambda_{0}
$$


where $\rho(n)$ is defined by (1.15) and $\lambda_{0}$ is the smaller root of the transcendental equation $\lambda=e^{\beta \lambda}$.

Lemma 6. Assume that (1.2) holds, $\rho(n)$ is defined by (1.15), $0<\beta \leq 1 / e$ and $x(n)$ is an eventually positive solution of $\left(E^{\prime}\right)$. Then

$$
\liminf _{n \rightarrow \infty} \frac{x(n-1)}{x(\rho(n))} \geq D(\beta)
$$

\section{MAIN RESULTS}

\subsection{RETARDED DIFFERENCE EQUATIONS}

Based on Lemmas 1, 2 and 3, we further study $(E)$ and derive new sufficient oscillation conditions, involving limsup, which improve on all previously known results in the literature.

Theorem 7. Assume that (1.1), (1.8) hold, and $h(n)$ is defined by (1.7). If for some $w \in \mathbb{N}$

$$
\limsup _{n \rightarrow \infty} \sum_{\ell=h(n)}^{n} \bar{P}(\ell) \exp \left(\sum_{j=\tau(\ell)}^{h(n)-1} \bar{P}(j) \prod_{i=\tau(j)}^{j-1} \frac{1}{1-\bar{R}_{w}(i)}\right)>1
$$

where

$$
\bar{R}_{w}(n)=\bar{P}(n)\left[1+\sum_{\ell=\tau(n)}^{n-1} \bar{P}(\ell) \exp \left(\sum_{j=\tau(\ell)}^{n-1} \bar{P}(j) \prod_{i=\tau(j)}^{j-1} \frac{1}{1-\bar{R}_{w-1}(i)}\right)\right]
$$

with $\bar{R}_{0}(n)=\lambda_{0} \bar{P}(n), \bar{P}(n)=\sum_{i=1}^{m} p_{i}(n)$ and $\lambda_{0}$ be the smaller root of the transcendental equation $\lambda=e^{\alpha \lambda}$, then all solutions of $(E)$ are oscillatory.

Proof. Assume, for the sake of contradiction, that there exists a nonoscillatory solution $(x(n))_{n \geq-v}$ of $(E)$. Since $(-x(n))_{n \geq-v}$ is also a solution of $(E)$, we can confine our discussion only to the case where $x(n)>0$, for all large $n$. Let $n_{1} \geq-v$ be an integer such that $x(n)>0$, for all $n \geq n_{1}$. Then, there exists $n_{2} \geq n_{1}$ such that $x\left(\tau_{i}(n)\right)>0$, for all $n \geq n_{2}$. In view of this, Eq. $(E)$ becomes

$$
\Delta x(n)=-\sum_{i=1}^{m} p_{i}(n) x\left(\tau_{i}(n)\right) \leq 0, \quad \text { for all } n \geq n_{2},
$$

which means that $(x(n))$ is an eventually nonincreasing sequence of positive numbers. 
Taking into account the fact that $\tau_{i}(n) \leq h_{i}(n) \leq h(n),(E)$ implies that

$$
\Delta x(n)+\left(\sum_{i=1}^{m} p_{i}(n)\right) x(h(n)) \leq 0,
$$

or

$$
\Delta x(n)+\bar{P}(n) x(h(n)) \leq 0, \quad n \geq n_{2} .
$$

On the other hand, by (2.2), for each $\epsilon>0$, there exists a $n(\epsilon)$ such that

$$
\frac{x(h(n))}{x(n)}>\lambda_{0}-\epsilon, \quad \text { for all } n \geq n(\epsilon) \geq n_{2} .
$$

Combining inequalities (3.3) and (3.4), we obtain

$$
\Delta x(n)+\left(\lambda_{0}-\epsilon\right) \bar{P}(n) x(n)<0, \quad n \geq n(\epsilon),
$$

or

$$
\Delta x(n)+\bar{R}_{0}(n, \epsilon) x(n)<0, \quad n \geq n(\epsilon),
$$

where

$$
\bar{R}_{0}(n, \epsilon)=\left(\lambda_{0}-\epsilon\right) \bar{P}(n) .
$$

Applying the discrete Grönwall inequality in (3.5), we have

$$
x(k)>x(n) \prod_{i=k}^{n-1} \frac{1}{1-\bar{R}_{0}(i, \epsilon)}, \quad \text { for all } n \geq k \geq n(\epsilon) .
$$

Now, dividing $(E)$ by $x(n)$ and summing up from $k$ to $n-1$, we get

$$
\sum_{j=k}^{n-1} \frac{\Delta x(j)}{x(j)}=-\sum_{j=k}^{n-1} \sum_{i=1}^{m} p_{i}(j) \frac{x\left(\tau_{i}(j)\right)}{x(j)}
$$

or

$$
\sum_{j=k}^{n-1} \frac{\Delta x(j)}{x(j)} \leq-\sum_{j=k}^{n-1}\left(\sum_{i=1}^{m} p_{i}(j)\right) \frac{x(\tau(j))}{x(j)}
$$

i.e.,

$$
\sum_{j=k}^{n-1} \frac{\Delta x(j)}{x(j)} \leq-\sum_{j=k}^{n-1} \bar{P}(j) \frac{x(\tau(j))}{x(j)} .
$$

Also, since $e^{x} \geq x+1, x>0$, we have

$$
\begin{aligned}
\sum_{j=k}^{n-1} \frac{\Delta x(j)}{x(j)} & =\sum_{j=k}^{n-1}\left(\frac{x(j+1)}{x(j)}-1\right) \\
& =\sum_{j=k}^{n-1}\left[\exp \left(\ln \frac{x(j+1)}{x(j)}\right)-1\right]
\end{aligned}
$$


DIFFERENCE EQUATIONS

$$
\begin{aligned}
& \geq \sum_{j=k}^{n-1}\left[\ln \frac{x(j+1)}{x(j)}+1-1\right] \\
& =\sum_{j=k}^{n-1} \ln \frac{x(j+1)}{x(j)}=\ln \frac{x(n)}{x(k)},
\end{aligned}
$$

or

$$
\sum_{j=k}^{n-1} \frac{\Delta x(j)}{x(j)} \geq \ln \frac{x(n)}{x(k)} .
$$

Combining inequalities (3.7) and (3.8), we obtain

$$
-\sum_{j=k}^{n-1} \bar{P}(j) \frac{x(\tau(j))}{x(j)} \geq \ln \frac{x(n)}{x(k)},
$$

or

$$
\ln \frac{x(k)}{x(n)} \geq \sum_{j=k}^{n-1} \bar{P}(j) \frac{x(\tau(j))}{x(j)} .
$$

Since $\tau(j)<j,(3.6)$ implies

$$
x(\tau(j))>x(j) \prod_{i=\tau(j)}^{j-1} \frac{1}{1-\bar{R}_{0}(i, \epsilon)} .
$$

In view of $(3.10),(3.9)$ gives

$$
\ln \frac{x(k)}{x(n)}>\sum_{j=k}^{n-1} \bar{P}(j) \prod_{i=\tau(j)}^{j-1} \frac{1}{1-\bar{R}_{0}(i, \epsilon)},
$$

or

$$
x(k)>x(n) \exp \left(\sum_{j=k}^{n-1} \bar{P}(j) \prod_{i=\tau(j)}^{j-1} \frac{1}{1-\bar{R}_{0}(i, \epsilon)}\right) .
$$

Summing up $(E)$ from $\tau(n)$ to $n-1$, we have

$$
x(n)-x(\tau(n))+\sum_{\ell=\tau(n)}^{n-1} \sum_{i=1}^{m} p_{i}(\ell) x\left(\tau_{i}(\ell)\right)=0,
$$

or

$$
x(n)-x(\tau(n))+\sum_{\ell=\tau(n)}^{n-1}\left(\sum_{i=1}^{m} p_{i}(\ell)\right) x(\tau(\ell)) \leq 0,
$$

i.e.,

$$
x(n)-x(\tau(n))+\sum_{\ell=\tau(n)}^{n-1} \bar{P}(\ell) x(\tau(\ell)) \leq 0 .
$$


Since $\tau(\ell) \leq h(\ell) \leq h(n)<n,(3.11)$ implies that

$$
x(\tau(\ell))>x(n) \exp \left(\sum_{j=\tau(\ell)}^{n-1} \bar{P}(j) \prod_{i=\tau(j)}^{j-1} \frac{1}{1-\bar{R}_{0}(i, \epsilon)}\right) .
$$

Combining (3.12) and (3.13), we have

$$
x(n)-x(\tau(n))+x(n) \sum_{\ell=\tau(n)}^{n-1} \bar{P}(\ell) \exp \left(\sum_{j=\tau(\ell)}^{n-1} \bar{P}(j) \prod_{i=\tau(j)}^{j-1} \frac{1}{1-\bar{R}_{0}(i, \epsilon)}\right)<0 .
$$

Multiplying the last inequality by $\bar{P}(n)$, we take

$$
\begin{aligned}
\bar{P}(n) x(n) & -\bar{P}(n) x(\tau(n)) \\
& +\bar{P}(n) x(n) \sum_{\ell=\tau(n)}^{n-1} \bar{P}(\ell) \exp \left(\sum_{j=\tau(\ell)}^{n-1} \bar{P}(j) \prod_{i=\tau(j)}^{j-1} \frac{1}{1-\bar{R}_{0}(i, \epsilon)}\right)<0 .
\end{aligned}
$$

Furthermore,

$$
\Delta x(n)=-\sum_{i=1}^{m} p_{i}(n) x\left(\tau_{i}(n)\right) \leq-x(\tau(n)) \sum_{i=1}^{m} p_{i}(n),
$$

i.e.,

$$
\Delta x(n) \leq-\bar{P}(n) x(\tau(n)) .
$$

In view of this, (3.14) gives

$$
\Delta x(n)+\bar{P}(n) x(n)+\bar{P}(n) x(n) \sum_{\ell=\tau(n)}^{n-1} \bar{P}(\ell) \exp \left(\sum_{j=\tau(\ell)}^{n-1} \bar{P}(j) \prod_{i=\tau(j)}^{j-1} \frac{1}{1-\bar{R}_{0}(i, \epsilon)}\right)<0,
$$

or

$$
\Delta x(n)+\bar{P}(n)\left[1+\sum_{\ell=\tau(n)}^{n-1} \bar{P}(\ell) \exp \left(\sum_{j=\tau(\ell)}^{n-1} \bar{P}(j) \prod_{i=\tau(j)}^{j-1} \frac{1}{1-\bar{R}_{0}(i, \epsilon)}\right)\right] x(n)<0 .
$$

Therefore

$$
\Delta x(n)+\bar{R}_{1}(n, \epsilon) x(n)<0,
$$

where

$$
\bar{R}_{1}(n, \epsilon)=\bar{P}(n)\left[1+\sum_{\ell=\tau(n)}^{n-1} \bar{P}(\ell) \exp \left(\sum_{j=\tau(\ell)}^{n-1} \bar{P}(j) \prod_{i=\tau(j)}^{j-1} \frac{1}{1-\bar{R}_{0}(i, \epsilon)}\right)\right] .
$$

Repeating the above argument, where (3.15) is used instead of (3.5), leads to a new estimate

$$
\Delta x(n)+\bar{R}_{2}(n, \epsilon) x(n)<0,
$$


where

$$
\bar{R}_{2}(n, \epsilon)=\bar{P}(n)\left[1+\sum_{\ell=\tau(n)}^{n-1} \bar{P}(\ell) \exp \left(\sum_{j=\tau(\ell)}^{n-1} \bar{P}(j) \prod_{i=\tau(j)}^{j-1} \frac{1}{1-\bar{R}_{1}(i, \epsilon)}\right)\right] .
$$

By induction, we get

$$
\Delta x(n)+\bar{R}_{w}(n, \epsilon) x(n)<0, \quad(w \in \mathbb{N})
$$

where

$$
\bar{R}_{w}(n, \epsilon)=\bar{P}(n)\left[1+\sum_{\ell=\tau(n)}^{n-1} \bar{P}(\ell) \exp \left(\sum_{j=\tau(\ell)}^{n-1} \bar{P}(j) \prod_{i=\tau(j)}^{j-1} \frac{1}{1-\bar{R}_{w-1}(i, \epsilon)}\right)\right]
$$

and

$$
x(\tau(\ell))>x(h(n)) \exp \left(\sum_{j=\tau(\ell)}^{h(n)-1} \bar{P}(j) \prod_{i=\tau(j)}^{j-1} \frac{1}{1-\bar{R}_{w}(i, \epsilon)}\right) .
$$

Summing up $(E)$ from $h(n)$ to $n$, we have

$$
x(n+1)-x(h(n))+\sum_{\ell=h(n)}^{n} \sum_{i=1}^{m} p_{i}(\ell) x\left(\tau_{i}(\ell)\right)=0,
$$

or

$$
x(n+1)-x(h(n))+\sum_{\ell=h(n)}^{n}\left(\sum_{i=1}^{m} p_{i}(\ell)\right) x(\tau(\ell)) \leq 0,
$$

i.e.,

$$
x(n+1)-x(h(n))+\sum_{\ell=h(n)}^{n} \bar{P}(\ell) x(\tau(\ell)) \leq 0 .
$$

Using (3.17), the last inequality gives

$$
\begin{aligned}
x(n+1)- & x(h(n)) \\
& +x(h(n)) \sum_{\ell=h(n)}^{n} \bar{P}(\ell) \exp \left(\sum_{j=\tau(\ell)}^{h(n)-1} \bar{P}(j) \prod_{i=\tau(j)}^{j-1} \frac{1}{1-\bar{R}_{w}(i, \epsilon)}\right)<0 .
\end{aligned}
$$

The inequality is still valid, if we omit the term $x(n+1)>0$, in the left-hand side:

$$
-x(h(n))+x(h(n)) \sum_{\ell=h(n)}^{n} \bar{P}(\ell) \exp \left(\sum_{j=\tau(\ell)}^{h(n)-1} \bar{P}(j) \prod_{i=\tau(j)}^{j-1} \frac{1}{1-\bar{R}_{w}(i, \epsilon)}\right)<0 .
$$

This implies

$$
x(h(n))\left[\sum_{\ell=h(n)}^{n} \bar{P}(\ell) \exp \left(\sum_{j=\tau(\ell)}^{h(n)-1} \bar{P}(j) \prod_{i=\tau(j)}^{j-1} \frac{1}{1-\bar{R}_{w}(i, \epsilon)}\right)-1\right]<0,
$$


i.e.,

$$
\limsup _{n \rightarrow \infty} \sum_{\ell=h(n)}^{n} \bar{P}(\ell) \exp \left(\sum_{j=\tau(\ell)}^{h(n)-1} \bar{P}(j) \prod_{i=\tau(j)}^{j-1} \frac{1}{1-\bar{R}_{w}(i, \epsilon)}\right) \leq 1 .
$$

Since $\epsilon$ may be taken arbitrarily small, this inequality contradicts (3.1).

The proof of the theorem is complete.

Theorem 8. Assume that (1.1), (1.8) hold, $h(n)$ is defined by (1.7) and $\alpha$ by (1.3), with $0<\alpha \leq 1 / e$. If for some $w \in \mathbb{N}$

$$
\limsup _{n \rightarrow \infty} \sum_{\ell=h(n)}^{n} \bar{P}(\ell) \exp \left(\sum_{j=\tau(\ell)}^{h(n)-1} \bar{P}(j) \prod_{i=\tau(j)}^{j-1} \frac{1}{1-\bar{R}_{w}(i)}\right)>1-D(\alpha)
$$

where $\bar{R}_{w}(n)$ is defined by (3.2), then all solutions of $(E)$ are oscillatory.

Proof. Assume, for the sake of contradiction, that $(x(n))_{n \geq-v}$ is a nonoscillatory solution of $(E)$. Since $(-x(n))_{n \geq-v}$ is also a solution of $(E)$, we can confine our discussion only to the case where $x(n)>0$, for all large $n$. Then, as in the proof of Theorem 7, for sufficiently large $n,(3.18)$ is satisfied, i.e.,

$$
x(n+1)-x(h(n))+x(h(n)) \sum_{\ell=h(n)}^{n} \bar{P}(\ell) \exp \left(\sum_{j=\tau(\ell)}^{h(n)-1} \bar{P}(j) \prod_{i=\tau(j)}^{j-1} \frac{1}{1-\bar{R}_{w}(i, \epsilon)}\right)<0 .
$$

That is,

$$
\sum_{\ell=h(n)}^{n} \bar{P}(\ell) \exp \left(\sum_{j=\tau(\ell)}^{h(n)-1} \bar{P}(j) \prod_{i=\tau(j)}^{j-1} \frac{1}{1-\bar{R}_{w}(i, \epsilon)}\right)<1-\frac{x(n+1)}{x(h(n))},
$$

which gives

$$
\limsup _{n \rightarrow \infty} \sum_{\ell=h(n)}^{n} \bar{P}(\ell) \exp \left(\sum_{j=\tau(\ell)}^{h(n)-1} \bar{P}(j) \prod_{i=\tau(j)}^{j-1} \frac{1}{1-\bar{R}_{w}(i, \epsilon)}\right) \leq 1-\liminf _{n \rightarrow \infty} \frac{x(n+1)}{x(h(n))} .
$$

Since $0<\alpha \leq 1 / e$, by Lemma 3, inequality (2.9) holds. So the last inequality leads to

$$
\limsup _{n \rightarrow \infty} \sum_{\ell=h(n)}^{n} \bar{P}(\ell) \exp \left(\sum_{j=\tau(\ell)}^{h(n)-1} \bar{P}(j) \prod_{i=\tau(j)}^{j-1} \frac{1}{1-\bar{R}_{w}(i, \epsilon)}\right) \leq 1-D(\alpha) .
$$

Since $\epsilon$ may be taken arbitrarily small, this inequality contradicts (3.19).

The proof of the theorem is complete.

Remark 9. It is clear that the left-hand sides of both conditions (3.1) and (3.19) are identical, also the right-hand side of condition (3.19) reduces to (3.1) in case that 
$\alpha=0$. So it seems that Theorem 8 is the same as Theorem 7 when $\alpha=0$. However, one may notice that condition $0<\alpha \leq 1 / e$ is required in Theorem 8 but not in Theorem 7 .

Theorem 10. Assume that (1.1), (1.8) hold, $h(n)$ is defined by (1.7) and $\alpha$ by (1.3), with $0<\alpha \leq 1 /$ e. If for some $w \in \mathbb{N}$

$$
\limsup _{n \rightarrow \infty} \sum_{\ell=h(n)}^{n} \bar{P}(\ell) \exp \left(\sum_{j=\tau(\ell)}^{n} \bar{P}(j) \prod_{i=\tau(j)}^{j-1} \frac{1}{1-\bar{R}_{w}(i)}\right)>\frac{1}{D(\alpha)}-1
$$

where $\bar{R}_{w}(n)$ is defined by (3.2), then all solutions of $(E)$ are oscillatory.

Proof. Assume, for the sake of contradiction, that $(x(n))_{n \geq-v}$ is an eventually positive solution of $(E)$. Then, as in the proof of Theorem 7, (3.17) is satisfied, i.e.,

$$
x(\tau(\ell))>x(h(n)) \exp \left(\sum_{j=\tau(\ell)}^{h(n)-1} \bar{P}(j) \prod_{i=\tau(j)}^{j-1} \frac{1}{1-\bar{R}_{w}(i, \epsilon)}\right) .
$$

Therefore, for a sufficiently large $n$, we have

$$
x(\tau(\ell))>x(n+1) \exp \left(\sum_{j=\tau(\ell)}^{n} \bar{P}(j) \prod_{i=\tau(j)}^{j-1} \frac{1}{1-\bar{R}_{w}(i, \epsilon)}\right) .
$$

Summing up $(E)$ from $h(n)$ to $n$, we have

$$
x(n+1)-x(h(n))+\sum_{\ell=h(n)}^{n} \sum_{i=1}^{m} p_{i}(\ell) x\left(\tau_{i}(\ell)\right)=0,
$$

or

$$
x(n+1)-x(h(n))+\sum_{\ell=h(n)}^{n}\left(\sum_{i=1}^{m} p_{i}(\ell)\right) x(\tau(\ell)) \leq 0,
$$

i.e.,

$$
x(n+1)-x(h(n))+\sum_{\ell=h(n)}^{n} \bar{P}(\ell) x(\tau(\ell)) \leq 0 .
$$

In view of (3.21), the last inequality gives

$$
x(n+1)-x(h(n))+\sum_{\ell=h(n)}^{n} \bar{P}(\ell) x(n+1) \exp \left(\sum_{j=\tau(\ell)}^{n} \bar{P}(j) \prod_{i=\tau(j)}^{j-1} \frac{1}{1-\bar{R}_{w}(i, \epsilon)}\right)<0,
$$

or

$$
x(n+1)-x(h(n))+x(h(n)) \sum_{\ell=h(n)}^{n} \bar{P}(\ell) \frac{x(n+1)}{x(h(n))} \exp \left(\sum_{j=\tau(\ell)}^{n} \bar{P}(j) \prod_{i=\tau(j)}^{j-1} \frac{1}{1-\bar{R}_{w}(i, \epsilon)}\right)<0,
$$


or

$x(n+1)+x(h(n))\left[\frac{x(n+1)}{x(h(n))} \sum_{\ell=h(n)}^{n} \bar{P}(\ell) \exp \left(\sum_{j=\tau(\ell)}^{n} \bar{P}(j) \prod_{i=\tau(j)}^{j-1} \frac{1}{1-\bar{R}_{w}(i, \epsilon)}\right)-1\right]<0$.

Thus, for all sufficiently large $n$, it holds

$$
\sum_{\ell=h(n)}^{n} \bar{P}(\ell) \exp \left(\sum_{j=\tau(\ell)}^{n} \bar{P}(j) \prod_{i=\tau(j)}^{j-1} \frac{1}{1-\bar{R}_{w}(i, \epsilon)}\right)<\frac{x(h(n))}{x(n+1)}-1
$$

Letting $n \rightarrow \infty$, we take

$$
\limsup _{n \rightarrow \infty} \sum_{\ell=h(n)}^{n} \bar{P}(\ell) \exp \left(\sum_{j=\tau(\ell)}^{n} \bar{P}(j) \prod_{i=\tau(j)}^{j-1} \frac{1}{1-\bar{R}_{w}(i, \epsilon)}\right) \leq \limsup _{n \rightarrow \infty} \frac{x(h(n))}{x(n+1)}-1 .
$$

Since $0<\alpha \leq 1 / e$, by Lemma 3 , inequality (2.9) holds. So the last inequality leads to

$$
\limsup _{n \rightarrow \infty} \sum_{\ell=h(n)}^{n} \bar{P}(\ell) \exp \left(\sum_{j=\tau(\ell)}^{n} \bar{P}(j) \prod_{i=\tau(j)}^{j-1} \frac{1}{1-\bar{R}_{w}(i, \epsilon)}\right) \leq \frac{1}{D(\alpha)}-1
$$

Since $\epsilon$ may be taken arbitrarily small, this inequality contradicts (3.20).

The proof of the theorem is complete.

Remark 11. If $R_{w}(n, \epsilon) \geq 1$, then (3.16) guarantees that all solutions of $(E)$ are oscillatory. In fact, (3.16) gives

$$
\Delta x(n)+x(n) \leq 0
$$

which means that $x(n+1) \leq 0$. This contradics $x(n)>0$, for all $n \geq n_{2}$. Thus, in Theorems 7, 8 and 10 we consider only the case $R_{w}(n)<1$. Another conclusion, that can be drawn from the above, is that if at some point through the iterative process, we get a value of $w$, for which $R_{w}(n) \geq 1$, then the process terminates, since in any case, all solutions of $(E)$ will be oscillatory. The value of $w$, that is the number of iterations, obviously, depends on the coefficients $p_{i}(n)$ and the form of the non-monotone arguments $\tau_{i}(n)$.

\subsection{ADVANCED DIFFERENCE EQUATIONS}

Based on Lemmas 4, 5 and 6, similar oscillation theorems for the (dual) advanced difference equation $\left(E^{\prime}\right)$ can be derived easily. The proofs of these theorems are omitted, since they are quite similar to the proofs for a retarded equation. 
Theorem 12. Assume that (1.2), (1.16) hold, and $\rho(n)$ is defined by (1.15). If for some $w \in \mathbb{N}$

$$
\limsup _{n \rightarrow \infty} \sum_{\ell=n}^{\rho(n)} \bar{Q}(\ell) \exp \left(\sum_{j=\rho(n)+1}^{\sigma(\ell)} \bar{Q}(j) \prod_{i=j+1}^{\sigma(j)} \frac{1}{1-\bar{\Phi}_{w}(i)}\right)>1,
$$

where

$$
\bar{\Phi}_{w}(n)=\bar{Q}(n)\left[1+\sum_{\ell=n+1}^{\sigma(n)} \bar{Q}(\ell) \exp \left(\sum_{j=n+1}^{\sigma(\ell)} \bar{Q}(j) \prod_{i=j+1}^{\sigma(j)} \frac{1}{1-\bar{\Phi}_{w-1}(i)}\right)\right]
$$

with $\bar{\Phi}_{0}(n)=\lambda_{0} \bar{Q}(n), \bar{Q}(n)=\sum_{i=1}^{m} q_{i}(n)$ and $\lambda_{0}$ be the smaller root of the transcendental equation $\lambda=e^{\beta \lambda}$, then all solutions of $\left(E^{\prime}\right)$ are oscillatory.

Theorem 13. Assume that (1.2), (1.16) hold, $\rho(n)$ is defined by (1.15) and $\beta$ by (1.4), with $0<\beta \leq 1 /$ e. If for some $w \in \mathbb{N}$

$$
\limsup _{n \rightarrow \infty} \sum_{\ell=n}^{\rho(n)} \bar{Q}(\ell) \exp \left(\sum_{j=\rho(n)+1}^{\sigma(\ell)} \bar{Q}(j) \prod_{i=j+1}^{\sigma(j)} \frac{1}{1-\bar{\Phi}_{w}(i)}\right)>1-D(\beta),
$$

where $\bar{\Phi}_{w}(n)$ is defined by (3.23), then all solutions of $\left(E^{\prime}\right)$ are oscillatory.

Theorem 14. Assume that (1.2) and (1.16) hold, $\rho(n)$ is defined by (1.15) and $\beta$ by (1.4), with $0<\beta \leq 1 /$ e. If for some $w \in \mathbb{N}$

$$
\limsup _{n \rightarrow \infty} \sum_{\ell=n}^{\rho(n)} \bar{Q}(\ell) \exp \left(\sum_{j=n}^{\sigma(\ell)} \bar{Q}(j) \prod_{i=j+1}^{\sigma(j)} \frac{1}{1-\bar{\Phi}_{w}(i)}\right)>\frac{1}{D(\beta)}-1,
$$

where $\bar{\Phi}_{w}(n)$ is defined by (3.23), then all solutions of $\left(E^{\prime}\right)$ are oscillatory.

Remark 15. Similar comments to those in Remark 11, can be made for Theorems 12,13 and 14, concerning equation $\left(E^{\prime}\right)$.

\subsection{DIFFERENCE INEQUALITIES}

Slightly modifying the proofs of Theorems 7, 8, 10 and 12-14, we can establish the following result on deviating difference inequalities.

Theorem 16. Assume that all conditions of Theorem 7 [12] or 8 [13] or 10 [14] hold. Then

(i) the retarded [advanced] difference inequality

$$
\Delta x(n)+\sum_{i=1}^{m} p_{i}(n) x\left(\tau_{i}(n)\right) \leq 0, \quad n \in \mathbb{N}_{0}\left[\nabla x(n)-\sum_{i=1}^{m} q_{i}(n) x\left(\sigma_{i}(n)\right) \geq 0, \quad n \in \mathbb{N}\right]
$$


has no eventually positive solutions;

(ii) the retarded [advanced] difference inequality

$$
\Delta x(n)+\sum_{i=1}^{m} p_{i}(n) x\left(\tau_{i}(n)\right) \geq 0, \quad n \in \mathbb{N}_{0}\left[\nabla x(n)-\sum_{i=1}^{m} q_{i}(n) x\left(\sigma_{i}(n)\right) \leq 0, n \in \mathbb{N}\right]
$$

has no eventually negative solutions.

\section{EXAMPLES AND COMMENTS}

The examples in this section illustrate how the conditions established in this paper imply oscillations, where the previously known conditions fail.

Example 17. Consider the retarded difference equation

$$
\Delta x(n)+\frac{29}{500} x\left(\tau_{1}(n)\right)+\frac{29}{1000} x\left(\tau_{2}(n)\right)+\frac{37}{2500} x\left(\tau_{3}(n)\right)=0, \quad n \in \mathbb{N}_{0},
$$

with (see Fig. 1, (a))

$$
\tau_{1}(n)=\left\{\begin{array}{ll}
n-4, & \text { if } n=5 \mu \\
n-1, & \text { if } n=5 \mu+1 \\
n-5, & \text { if } n=5 \mu+2 \\
n-3, & \text { if } n=5 \mu+3 \\
n-6, & \text { if } n=5 \mu+4
\end{array} \quad \text { and } \quad \begin{array}{l}
\tau_{2}(n)=\tau_{1}(n)-1 \\
\tau_{3}(n)=\tau_{1}(n)-2
\end{array}\right.
$$

where $\mu \in \mathbb{N}_{0}$ and $\mathbb{N}_{0}$ is the set of nonnegative integers.

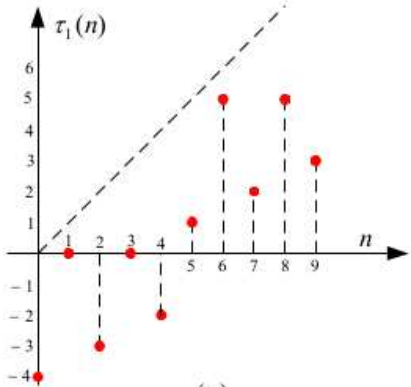

(a)

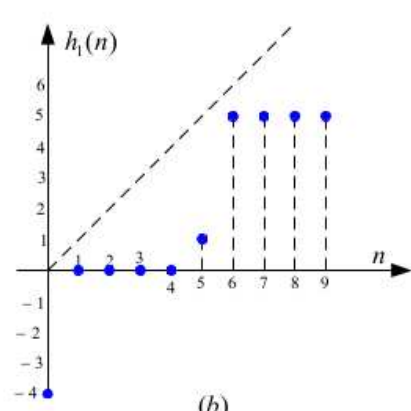

(b)

Figure 1: The graphs of $\tau_{1}(n)$ and $h_{1}(n)$ 
By (1.7), we see (Fig. 1, (b)) that

$$
h_{1}(n)=\left\{\begin{array}{ll}
n-4, & \text { if } n=5 \mu \\
n-1, & \text { if } n=5 \mu+1 \\
n-2, & \text { if } n=5 \mu+2 \\
n-3, & \text { if } n=5 \mu+3 \\
n-4, & \text { if } n=5 \mu+4
\end{array} \quad \text { and } \quad \begin{array}{l}
h_{2}(n)=h_{1}(n)-1 \\
h_{3}(n)=h_{1}(n)-2
\end{array} .\right.
$$

Consequently

$$
h(n)=\max _{1 \leq i \leq 3}\left\{h_{i}(n)\right\}=h_{1}(n) \quad \text { and } \quad \tau(n)=\max _{1 \leq i \leq 3} \tau_{i}(n)=\tau_{1}(n) .
$$

It is easy to see that

$$
\begin{aligned}
\alpha & =\liminf _{n \rightarrow \infty} \sum_{i=1}^{3} \sum_{j=\tau(n)}^{n-1} p_{i}(j)=\liminf _{\mu \rightarrow \infty} \sum_{i=1}^{3} \sum_{j=5 \mu}^{5 \mu} p_{i}(j) \\
& =\frac{29}{500}+\frac{29}{1000}+\frac{37}{2500}=\frac{509}{5000}=0.1018
\end{aligned}
$$

and the smaller root of equation $e^{\alpha \lambda}=\lambda$ is $\lambda_{0} \simeq 1.12087$.

Also, $\sum_{i=1}^{3} p_{i}(n)=0.1018<1$, i.e., (1.8) is satisfied for all $n \geq 0$.

Observe that the function $F_{\ell}: \mathbb{N}_{0} \rightarrow \mathbb{R}_{+}$defined as

$$
F_{w}(n)=\sum_{\ell=h(n)}^{n} \bar{P}(\ell) \exp \left(\sum_{j=\tau(\ell)}^{h(n)-1} \bar{P}(j) \prod_{i=\tau(j)}^{j-1} \frac{1}{1-\bar{R}_{w}(i)}\right)
$$

attains its maximum at $n=5 \mu, \mu \in \mathbb{N}_{0}$, for every $w \in \mathbb{N}$. Specifically,

$$
F_{1}(5 \mu)=\sum_{\ell=5 \mu-4}^{5 \mu} \bar{P}(\ell) \exp \left(\sum_{j=\tau(\ell)}^{5 \mu-5} \bar{P}(j) \prod_{i=\tau(j)}^{j-1} \frac{1}{1-\bar{R}_{1}(i)}\right)
$$

with

$$
\bar{R}_{1}(i)=\bar{P}(i)\left[1+\sum_{u=\tau(i)}^{i-1} \bar{P}(u) \exp \left(\sum_{v=\tau(u)}^{i-1} \bar{P}(v) \prod_{\xi=\tau(v)}^{v-1} \frac{1}{1-\lambda_{0} \bar{P}(\xi)}\right)\right]
$$

By using an algorithm on MATLAB software, we obtain

$$
F_{1}(5 \mu) \simeq 1.0066
$$

and therefore

$$
\limsup _{n \rightarrow \infty} F_{1}(n) \simeq 1.0066>1 .
$$

That is, condition (3.1) of Theorem 7 is satisfied, for $w=1$. Therefore all solutions of (4.1) are oscillatory. 
Observe, however, that

$$
\begin{aligned}
& \alpha=0.1018<\frac{1}{e} \\
& M D=\limsup _{\mu \rightarrow \infty} \sum_{i=1}^{3} \sum_{j=5 \mu-4}^{5 \mu} p_{i}(j) \\
& =5 \cdot\left(\frac{29}{500}+\frac{29}{1000}+\frac{37}{2500}\right)=0.509<1 \text {, } \\
& \sum_{j=5 \mu-4}^{5 \mu} \sum_{i=1}^{3} p_{i}(j) a_{1}^{-1}\left(5 \mu-4, \tau_{i}(j)\right) \\
& =\frac{29}{500} a_{1}^{-1}(5 \mu-4,5 \mu-5)+\frac{29}{1000} a_{1}^{-1}(5 \mu-4,5 \mu-6)+\frac{37}{2500} a_{1}^{-1}(5 \mu-4,5 \mu-7) \\
& +\frac{29}{500} a_{1}^{-1}(5 \mu-4,5 \mu-8)+\frac{29}{1000} a_{1}^{-1}(5 \mu-4,5 \mu-9)+\frac{37}{2500} a_{1}^{-1}(5 \mu-4,5 \mu-10) \\
& +\frac{29}{500} a_{1}^{-1}(5 \mu-4,5 \mu-5)+\frac{29}{1000} a_{1}^{-1}(5 \mu-4,5 \mu-6)+\frac{37}{2500} a_{1}^{-1}(5 \mu-4,5 \mu-7) \\
& +\frac{29}{500} a_{1}^{-1}(5 \mu-4,5 \mu-7)+\frac{29}{1000} a_{1}^{-1}(5 \mu-4,5 \mu-8)+\frac{37}{2500} a_{1}^{-1}(5 \mu-4,5 \mu-9) \\
& +\frac{29}{500} a_{1}^{-1}(5 \mu-4,5 \mu-4)+\frac{29}{1000} a_{1}^{-1}(5 \mu-4,5 \mu-5)+\frac{37}{2500} a_{1}^{-1}(5 \mu-4,5 \mu-6) \\
& =\frac{29}{500} \frac{1}{1-0.1018}+\frac{29}{1000} \frac{1}{(1-0.1018)^{2}}+\frac{37}{2500} \frac{1}{(1-0.1018)^{3}} \\
& +\frac{29}{500} \frac{1}{(1-0.1018)^{4}}+\frac{29}{1000} \frac{1}{(1-0.1018)^{5}}+\frac{37}{2500} \frac{1}{(1-0.1018)^{6}} \\
& +\frac{29}{500} \frac{1}{1-0.1018}+\frac{29}{1000} \frac{1}{(1-0.1018)^{2}}+\frac{37}{2500} \frac{1}{(1-0.1018)^{3}} \\
& +\frac{29}{500} \frac{1}{(1-0.1018)^{3}}+\frac{29}{1000} \frac{1}{(1-0.1018)^{4}}+\frac{37}{2500} \frac{1}{(1-0.1018)^{5}} \\
& +\frac{29}{500} \cdot 1+\frac{29}{1000} \frac{1}{1-0.1018}+\frac{37}{2500} \frac{1}{(1-0.1018)^{2}} \simeq 0.6973346 \text {. }
\end{aligned}
$$

Thus,

$$
\limsup _{n \rightarrow \infty} \sum_{j=h(n)}^{n} \sum_{i=1}^{3} p_{i}(j) a_{1}^{-1}\left(h(n), \tau_{i}(j)\right) \simeq 0.6973346<1 .
$$

Finally, by a MATLAB program, we obtain

$$
\begin{aligned}
\limsup _{n \rightarrow \infty} \sum_{j=h(n)}^{n} \mathcal{P}(j) \prod_{i=\tau(j)}^{h(n)-1} \frac{1}{1-\mathcal{P}_{1}(i)} & =\limsup _{\mu \rightarrow \infty} \sum_{j=5 \mu-4}^{5 \mu} \mathcal{P}(j) \prod_{i=\tau(j)}^{5 \mu-5} \frac{1}{1-\mathcal{P}_{1}(i)} \\
& \simeq 0.7321<1
\end{aligned}
$$


and

$$
\begin{aligned}
\limsup _{n \rightarrow \infty} \sum_{\ell=h(n)}^{n} \bar{P}(\ell) \prod_{i=\tau(\ell)}^{h(n)-1} \frac{1}{1-\bar{P}_{1}(i)} & =\limsup _{\mu \rightarrow \infty} \sum_{j=5 \mu-4}^{5 \mu} \bar{P}(\ell) \prod_{i=\tau(\ell)}^{5 \mu-5} \frac{1}{1-\bar{P}_{1}(i)} \\
& \simeq 0.8915<1
\end{aligned}
$$

That is, none of conditions (1.5), (1.6), (1.9) (for $r=1$ ), (1.10) (for $\ell=1$ ) and (1.12) (for $w=1$ ) is satisfied.

Comments. It is worth noting that condition (3.1) achieves a significant improvement, approximately $97.76 \%$, over the corresponding condition (1.6). We get that estimate by comparing the values on the left-side of these conditions. The improvement over the conditions (1.9), (1.10) and (1.12) is very satisfactory, around $44.35 \%, 37.49 \%$ and $12.91 \%$, respectively.

Finally, observe that conditions (1.9), (1.10) and (1.12) do not lead to oscillation, from the first iteration. On the contrary, condition (3.1) is satisfied from the first iteration. This means that our condition is better and much faster than (1.9), (1.10) and (1.12).

Example 18. Consider the advanced difference equation

$$
\nabla x(n)-\frac{79}{1000} x\left(\sigma_{1}(n)\right)-\frac{39}{1000} x\left(\sigma_{2}(n)\right)-\frac{19}{1000} x\left(\sigma_{2}(n)\right)=0, \quad n \in \mathbb{N},
$$

with (see Fig. 2, (a))

$$
\sigma_{1}(n)=\left\{\begin{array}{ll}
n+5, & \text { if } n=7 \mu+1 \\
n+1, & \text { if } n=7 \mu+2 \\
n+2, & \text { if } n=7 \mu+3 \\
n+1, & \text { if } n=7 \mu+4 \\
n+4, & \text { if } n=7 \mu+5 \\
n+2, & \text { if } n=7 \mu+6 \\
n+1, & \text { if } n=7 \mu+7
\end{array} \quad \text { and } \quad \sigma_{2}(n)=\sigma_{1}(n)+1\right.
$$

where $\mu \in \mathbb{N}_{0}$ and $\mathbb{N}_{0}$ is the set of non-negative integers. By (1.15), we see (Fig. 2, (b)) that

$$
\rho_{1}(n)=\left\{\begin{array}{ll}
n+2, & \text { if } n=7 \mu+1 \\
n+1, & \text { if } n=7 \mu+2 \\
n+2, & \text { if } n=7 \mu+3 \\
n+1, & \text { if } n=7 \mu+4 \\
n+3, & \text { if } n=7 \mu+5 \\
n+2, & \text { if } n=7 \mu+6 \\
n+1, & \text { if } n=7 \mu+7
\end{array} \quad \text { and } \quad \rho_{2}(n)=\rho_{1}(n)+1\right.
$$




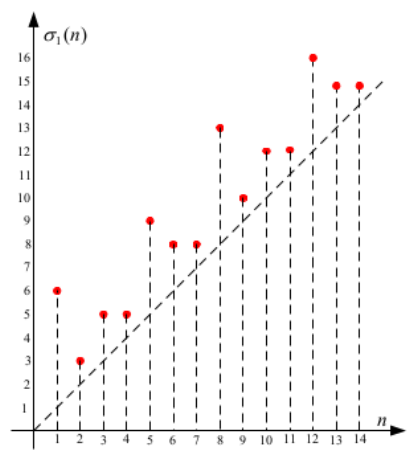

(a)

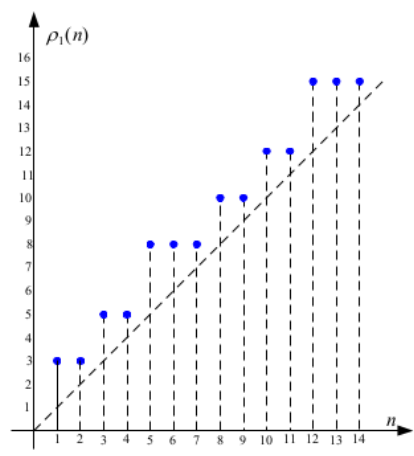

(b)

Figure 2: The graphs of $\sigma_{1}(n)$ and $\rho_{1}(n)$

Consequently,

$$
\rho(n)=\min _{1 \leq i \leq 3}\left\{\rho_{i}(n)\right\}=\rho_{1}(n) \quad \text { and } \quad \sigma(n)=\min _{1 \leq i \leq 3} \sigma_{i}(n)=\sigma_{1}(n) .
$$

It is easy to see that

$$
\begin{aligned}
\beta & =\liminf _{n \rightarrow \infty} \sum_{i=1}^{3} \sum_{j=n+1}^{\sigma(n)} q_{i}(j)=\liminf _{\mu \rightarrow \infty} \sum_{i=1}^{3} \sum_{j=7 \mu+3}^{7 \mu+3} q_{i}(j) \\
& =\frac{79}{1000}+\frac{39}{1000}+\frac{19}{1000}=0.137
\end{aligned}
$$

and the smaller root of equation $e^{\beta \lambda}=\lambda$ is $\lambda_{0} \simeq 1.17459$.

Also, $\sum_{i=1}^{3} q_{i}(n)=0.137<1$, i.e., (1.16) is satisfied for all $n \geq 1$.

We observe that the function $F: \mathbb{N}_{0} \rightarrow \mathbb{R}_{+}$defined as

$$
F_{w}(n)=\sum_{\ell=n}^{\rho(n)} \bar{Q}(\ell) \exp \left(\sum_{j=\rho(n)+1}^{\sigma(\ell)} \bar{Q}(j) \prod_{i=j+1}^{\sigma(j)} \frac{1}{1-\bar{\Phi}_{w}(i)}\right)
$$

with $\bar{Q}(n)=\sum_{i=1}^{3} q_{i}(n)=0.137$, attains its maximum at $n=7 \mu+5, \mu \in \mathbb{N}_{0}$, for every $w \in \mathbb{N}$. Specifically,

$$
F_{1}(7 \mu+5)=\sum_{\ell=7 \mu+5}^{7 \mu+8} \bar{Q}(\ell) \exp \left(\sum_{j=7 \mu+5}^{\sigma(\ell)} \bar{Q}(j) \prod_{i=j+1}^{\sigma(j)} \frac{1}{1-\bar{\Phi}_{1}(i)}\right)
$$

with

$$
\bar{\Phi}_{w}(i)=\bar{Q}(i)\left[1+\sum_{u=i+1}^{\sigma(i)} \bar{Q}(u) \exp \left(\sum_{v=i+1}^{\sigma(u)} \bar{Q}(v) \prod_{\xi=v+1}^{\sigma(v)} \frac{1}{1-\lambda_{0} \bar{Q}(\xi)}\right)\right]
$$


By using an algorithm on MATLAB software, we obtain

$$
F_{1}(7 \mu+5) \simeq 1.0230
$$

Thus

$$
\limsup _{n \rightarrow \infty} F_{1}(n) \simeq 1.0230>1 .
$$

That is, condition (3.22) of Theorem 12 is satisfied for $w=1$. Therefore, all solutions of (4.2) are oscillatory.

Observe, however, that

$$
\begin{aligned}
M A & =\limsup _{n \rightarrow \infty} \sum_{i=1}^{3} \sum_{j=7 \mu+5}^{7 \mu+8} q_{i}(j) \\
& =4 \cdot\left(\frac{79}{1000}+\frac{39}{1000}+\frac{19}{1000}\right) \simeq 0.548<1 .
\end{aligned}
$$

Also,

$$
\begin{aligned}
& \sum_{j=7 \mu+5}^{7 \mu+8} \sum_{i=1}^{3} q_{i}(j) b_{1}^{-1}\left(7 \mu+8, \sigma_{i}(j)\right) \\
& =\frac{79}{1000}\left[\begin{array}{c}
b_{1}^{-1}\left(7 \mu+8, \sigma_{1}(7 \mu+5)\right)+b_{1}^{-1}\left(7 \mu+8, \sigma_{1}(7 \mu+6)\right) \\
+b_{1}^{-1}\left(7 \mu+8, \sigma_{1}(7 \mu+7)\right)+b_{1}^{-1}\left(7 \mu+8, \sigma_{1}(7 \mu+8)\right)
\end{array}\right] \\
& +\frac{39}{1000}\left[\begin{array}{c}
b_{1}^{-1}\left(7 \mu+8, \sigma_{2}(7 \mu+5)\right)+b_{1}^{-1}\left(7 \mu+8, \sigma_{2}(7 \mu+6)\right) \\
+b_{1}^{-1}\left(7 \mu+8, \sigma_{2}(7 \mu+7)\right)+b_{1}^{-1}\left(7 \mu+8, \sigma_{2}(7 \mu+8)\right)
\end{array}\right] \\
& +\frac{19}{1000}\left[\begin{array}{c}
b_{1}^{-1}\left(7 \mu+8, \sigma_{3}(7 \mu+5)\right)+b_{1}^{-1}\left(7 \mu+8, \sigma_{3}(7 \mu+6)\right) \\
+b_{1}^{-1}\left(7 \mu+8, \sigma_{3}(7 \mu+7)\right)+b_{1}^{-1}\left(7 \mu+8, \sigma_{3}(7 \mu+8)\right)
\end{array}\right] \\
& =\frac{79}{1000}\left[\begin{array}{c}
b_{1}^{-1}(7 \mu+8,7 \mu+9)+b_{1}^{-1}(7 \mu+8,7 \mu+8) \\
+b_{1}^{-1}(7 \mu+8,7 \mu+8)+b_{1}^{-1}(7 \mu+8,7 \mu+13)
\end{array}\right] \\
& +\frac{39}{1000}\left[\begin{array}{c}
b_{1}^{-1}(7 \mu+8,7 \mu+10)+b_{1}^{-1}(7 \mu+8,7 \mu+9) \\
+b_{1}^{-1}(7 \mu+8,7 \mu+9)+b_{1}^{-1}(7 \mu+8,7 \mu+14)
\end{array}\right] \\
& +\frac{19}{1000}\left[\begin{array}{c}
b_{1}^{-1}(7 \mu+8,7 \mu+11)+b_{1}^{-1}(7 \mu+8,7 \mu+10) \\
+b_{1}^{-1}(7 \mu+8,7 \mu+10)+b_{1}^{-1}(7 \mu+8,7 \mu+15)
\end{array}\right] \\
& =\frac{79}{1000}\left[\frac{1}{1-0.137}+1+1+\frac{1}{(1-0.137)^{5}}\right] \\
& +\frac{39}{1000}\left[\frac{1}{(1-0.137)^{2}}+2 \cdot \frac{1}{1-0.137}+\frac{1}{(1-0.137)^{6}}\right] \\
& +\frac{19}{1000}\left[\frac{1}{(1-0.137)^{3}}+2 \cdot \frac{1}{(1-0.137)^{2}}+\frac{1}{(1-0.137)^{7}}\right]
\end{aligned}
$$




$$
\simeq 0.7856
$$

Therefore

$$
\limsup _{n \rightarrow \infty} \sum_{j=n}^{\rho(n)} \sum_{i=1}^{3} q_{i}(j) b_{1}^{-1}\left(\rho(n), \sigma_{i}(j)\right) \simeq 0.7856<1 .
$$

Finally, by using algorithms on MATLAB software, we obtain

$$
\limsup _{n \rightarrow \infty} \sum_{j=n}^{\rho(n)} Q(j) \prod_{i=\rho(n)+1}^{\sigma(j)} \frac{1}{1-Q_{1}(i)} \simeq 0.8347<1
$$

and

$$
\limsup _{n \rightarrow \infty} \sum_{\ell=n}^{\rho(n)} \bar{Q}(\ell) \prod_{i=\rho(n)+1}^{\sigma(\ell)} \frac{1}{1-\bar{Q}_{1}(i)} \simeq 0.9709<1 .
$$

That is, none of conditions (1.14), (1.17) (for $r=1$ ), (1.18) (for $\ell=1$ ) and (1.20) (for $w=1)$ is satisfied.

Comments. It is worth noting that condition (3.22) achieves a significant improvement, approximately $86.68 \%$, over the corresponding condition (1.14). We get that estimate by comparing the values on the left-side of these conditions. The improvement over the conditions (1.17), (1.18) and (1.20) is very satisfactory, around $30.22 \%, 22.56 \%$ and $5.37 \%$, respectively.

Finally, observe that conditions (1.17), (1.18) and (1.20) do not lead to oscillation from the first iteration. On the contrary, condition (3.22) is satisfied from the first iteration. This means that our condition is better and much faster than (1.17), (1.18) and (1.20).

Remark 19. Similarly, one can construct examples to illustrate the other main results.

\section{ACKNOWLEDGEMENTS}

The work on this research has been supported by the internal grant project no. FEI2017-39.

\section{REFERENCES}

[1] L. Berezansky and E. Braverman, On existence of positive solutions for linear difference equations with several delays, Advances Dyn. Syst. Appl., 1 (2006), $29-47$. 
[2] E. Braverman, G. E. Chatzarakis and I. P. Stavroulakis, Iterative oscillation tests for difference equations with several non-monotone arguments, J. Difference Equ. Appl., 21 (2015), no. 9, 854-874.

[3] G. E. Chatzarakis, L. Horvat-Dmitrović and M. Paš ić, Oscillation tests for Difference Equations with several non-monotone deviating arguments, Math. Slovaca (2018), In Press.

[4] G. E. Chatzarakis and I. Jadlovská, Oscillations in difference equations with several arguments using an iterative method, Filomat, 32, no. 1 (2018), 255-273.

[5] G. E. Chatzarakis, T. Kusano and I. P. Stavroulakis, Oscillation conditions for difference equations with several variable arguments, Math. Bohem., 140 (2015), No. 3, 291-311.

[6] G. E. Chatzarakis, J. Manojlovic, S. Pinelas and I. P. Stavroulakis, Oscillation criteria of difference equations with several deviating arguments, Yokohama Math. J., 60 (2014), 13-31.

[7] G.E. Chatzarakis, Ch. G. Philos and I.P. Stavroulakis, Oscillations of first order linear difference equations with general delay argument, Portugal. Math., 66 (2009), 513-533.

[8] G. E. Chatzarakis, S. Pinelas and I. P. Stavroulakis, Oscillations of difference equations with several deviated arguments, Aequat. Math., 88 (2014), 105-123.

[9] L. H. Erbe, Q. K. Kong and B.G. Zhang, Oscillation Theory for Functional Differential Equations, Marcel Dekker, New York, 1995.

[10] X. Li and D. Zhu, Oscillation of advanced difference equations with variable coefficients, Ann. Differential Equations, 18 (2002), 254-263.

[11] X. N. Luo, Y. Zhou and C. F. Li, Oscillations of a nonlinear difference equation with several delays, Mathematica Bohemica, 128 (2003), 309-317.

[12] X. H. Tang and J. S. Yu, Oscillation of delay difference equations, Comput. Math. Appl., 37 (1999), 11-20.

[13] X. H. Tang and R. Y. Zhang, New oscillation criteria for delay difference equations, Comput. Math. Appl., 42 (2001), 1319-1330.

[14] X. Wang, Oscillation of delay difference equations with several delays, J. Math. Anal. Appl.286 (2003), 664-674.

[15] X. P. Wu and L. Wang, Zero-Hopf bifurcation analysis in delayed differential equations with two delays, J. Frankl. Inst. 354 (2017), 1484-1513.

[16] W. Yan, Q. Meng and J. Yan, Oscillation criteria for difference equation of variable delays, DCDIS Proceedings3 (2005), 641-647. 
[17] B. G. Zhang and C. J. Tian, Nonexistence and existence of positive solutions for difference equations with unbounded delay, Comput. Math. Appl., 36 (1998), 1-8.

[18] B. G. Zhang and Y. Zhou, Oscillations of difference equations with several delays, Comput. Math. Appl., 44 (2002), 817-821. 